\title{
Nichols algebras over groups with finite root system of rank two II
}

\author{
István Heckenberger and Leandro Vendramin \\ Communicated by Gunter Malle
}

\begin{abstract}
We classify all non-abelian groups $G$ for which there exists a pair $(V, W)$ of absolutely simple Yetter-Drinfeld modules over $G$ such that the Nichols algebra of the direct sum of $V$ and $W$ is finite-dimensional, under two assumptions: the square of the braiding between $V$ and $W$ is not the identity, and $G$ is generated by the support of $V$ and $W$. As a corollary, we prove that the dimensions of such $V$ and $W$ are at most six. As a tool we use the Weyl groupoid of $(V, W)$.
\end{abstract}

\section{Introduction}

In the theory of Hopf algebras, deep structure results have been achieved since the introduction of the Lifting Method of Andruskiewitsch and Schneider [5]. The aim of the method is to classify (finite-dimensional) pointed Hopf algebras. The idea of it is to generalize Lusztig's approach to quantum groups [21].

The Lifting Method is based on the understanding of the structure theory of certain braided Hopf algebras which are known as Nichols algebras. In Lusztig's setting this is the algebra $\mathbf{f}$, also known as $U_{q}\left(\mathfrak{n}_{+}\right)$. Motivated by the first classification results of finite-dimensional Nichols algebras of diagonal type [6,23], a complete solution was obtained by the first author [14]. The tool for the latter classification was the Weyl groupoid and the root system of a Nichols algebra of diagonal type, which was discovered in [13] using the theory of Lyndon words and PBW bases [20]. The Weyl groupoid was also used by Angiono to determine the defining relations of finite-dimensional Nichols algebras of diagonal type [8]. These results have far reaching consequences in the theory of Hopf algebras such as the classification of finite-dimensional pointed Hopf algebras with abelian coradical of order coprime to 210 (see [7]), and the proof of the AndruskiewitschSchneider conjecture for pointed Hopf algebras with abelian coradical [9].

In order to understand the structure of Nichols algebras of non-diagonal type, the Weyl groupoid of a Nichols algebra of diagonal type was generalized further ship. Leandro Vendramin was supported by Conicet and the Alexander von Humboldt Foundation. 
in several successive papers such as [4], [19], [16] and [15]. The first applications of this generalization were powerful enough to study pointed Hopf algebras in some cases where the coradical is a finite simple group [1,2]. The difficulties in extending these results, and scientific curiosity, ask for a better understanding of finite-dimensional Nichols algebras of semisimple Yetter-Drinfeld modules over arbitrary groups.

In [15] H.-J. Schneider and the first author introduced a method to study the Weyl groupoid of a Nichols algebra over a Hopf algebra with invertible antipode. The main achievement of the paper was a description of $(\operatorname{ad} V)^{n}(W)$, for two Yetter-Drinfeld modules $V, W$, in terms of the braiding. Also, a family $\left(\Gamma_{n}\right)_{n \geq 2}$ of groups was introduced as candidates admitting finite-dimensional Nichols algebras, and the finite-dimensional Nichols algebras over $\Gamma_{2}$ with finite root system of rank two were determined.

Roughly speaking, in this paper we prove that any non-abelian group $G$ having a finite-dimensional Nichols algebra with an irreducible finite root system of rank two has to be an epimorphic image of $\Gamma_{2}, \Gamma_{3}, \Gamma_{4}$ or another group $T$. As a corollary, we obtain that the dimension of the subspace of primitive elements of such a Nichols algebra has dimension at most 12. For the precise statement we refer to Theorem 4.5 and Corollary 4.6. These claims are expected to become very useful in different ways. For example, the study of Nichols algebras of tuples of irreducible Yetter-Drinfeld modules usually requires a good understanding of the rank two case. Further, our results combined with the methods in $[1,2]$ can be used to obtain strong restrictions on the support of an irreducible Yetter-Drinfeld module with finite-dimensional Nichols algebra over a group.

In order to obtain more precise claims on Nichols algebras over $G$, one has to perform detailed calculations about $(\operatorname{ad} V)^{m}(W)$ and $(\operatorname{ad} W)^{m}(V), m \geq 1$, as in [15, Section 4]. These calculations lead then to the classification of finitedimensional Nichols algebras with finite root system of rank two, see $[17,18]$.

Our method is based on the Weyl groupoid. Let $V, W$ be absolutely simple Yetter-Drinfeld modules over $G$ such that the pair $(V, W)$ admits all reflections and the Weyl groupoid $\mathcal{W}(V, W)$ is finite. By [4, Theorem 3.12, Proposition 3.23], this is the case if the Nichols algebra of $V \oplus W$ is finite-dimensional. We prove that there exists an object of $\mathcal{W}(V, W)$ which has a Cartan matrix of finite type. Thus we have to analyze the consequences of $(\operatorname{ad} V)^{2}(W)=0,(\operatorname{ad} W)^{4}(V)=0$. We obtain restrictions regarding decomposability, size and further information on supp $V$ and $\operatorname{supp} W$. In particular, Theorem 4.4 tells that for a pair $(V, W)$ of Yetter-Drinfeld modules over $G$ such that $(\operatorname{ad} V)(W) \neq 0,(\operatorname{ad} V)^{2}(W)=0$ and $(\operatorname{ad} W)^{4}(V)=0$, it must be the case that $\operatorname{supp} V \cup \operatorname{supp} W$ is isomorphic to one of five quandles, all of size at most six. Our results are based on Proposition 5.5 claiming the non-vanishing of $(\operatorname{ad} V)^{m+1}(W)$ under some assumptions 
on the structure of $\operatorname{supp} V$ and $\operatorname{supp} W$. It is an interesting fact that for this proposition and for many of its consequences we do not need to assume that $V$ and $W$ have finite support or that their supports are conjugacy classes. Therefore Proposition 5.5 and its consequences can also be used to deal with Nichols algebras of arbitrary tuples of irreducible Yetter-Drinfeld modules over groups.

The structure of the paper is as follows. First we recall some facts on groups with abelian centralizers, quandles and their enveloping groups in Sections 1 and 2. In Section 3 we prove with Corollary 3.2 that connected Weyl groupoids of rank two admitting a finite irreducible root system have an object with a Cartan matrix of finite type. Section 4 is devoted to the study of Nichols algebras over groups. After discussing some technicalities, we formulate our main results, Theorem 4.5 and Corollary 4.6. In Section 5 we give a step-by-step proof of Theorem 4.4.

\section{Preliminaries}

\subsection{Groups with abelian centralizers}

Recall from [22] that a group has abelian centralizers if the centralizer of every non-central element is abelian. The following definition goes back to Hall [12].

Definition 1.1. Let $G$ and $H$ be two groups. We say that $G$ is isoclinic to $H$ if there exist isomorphisms $\zeta: G / Z(G) \rightarrow H / Z(H)$ and $\eta:[G, G] \rightarrow[H, H]$ such that if $g_{1}, g_{2} \in G, h_{1}, h_{2} \in H$, and $\zeta\left(g_{i} Z(G)\right)=h_{i} Z(H)$ for $i=1,2$, then $\eta\left[g_{1}, g_{2}\right]=\left[h_{1}, h_{2}\right]$. In this case we write $G \sim H$.

It is clear that the relation of isoclinism is an equivalence relation. The following lemma is due to Hall [12, p. 134].

Lemma 1.2. Let $G$ be a group and $K \triangleleft G$. The following hold:

(1) $G / K \sim G /(K \cap[G, G])$.

(2) If $K \subseteq[G, G]$ and $G \sim H$ for some group $H$ via the maps $\zeta$ and $\eta$, then $\eta(K) \triangleleft H$ and $G / K \sim H / \eta(K)$.

The following lemma was proved in [22, Lemma 3.4]. For completeness we give a proof in the context of this paper.

Lemma 1.3. Let $G$ and $H$ be groups and assume that $G \sim H$. If $G$ has abelian centralizers, then $H$ has abelian centralizers.

Proof. Let $h \in H \backslash Z(H)$ and let $h_{1}, h_{2} \in H^{h}$. Since $G \sim H$, there exist elements $g, g_{1}, g_{2} \in G$ such that $\zeta(g Z(G))=h Z(H)$ and $\zeta\left(g_{i} Z(G)\right)=h_{i} Z(H)$ 
for $i=1$, 2. Further $g \notin Z(G)$ since $h \notin Z(H)$. Then

$$
1=\left[h, h_{i}\right]=\eta\left[g, g_{i}\right]
$$

and hence $g_{i} \in G^{g}$. Therefore

$$
1=\eta\left[g_{1}, g_{2}\right]=\left[h_{1}, h_{2}\right]
$$

and $H^{h}$ is abelian.

\subsection{Quandles}

Recall that a quandle is a non-empty set $X$ with a binary operation $\triangleright$ such that

- the map $\varphi_{i}: X \rightarrow X, j \mapsto i \triangleright j$, is bijective for all $i \in X$,

- $i \triangleright(j \triangleright k)=(i \triangleright j) \triangleright(i \triangleright k)$ for all $i, j, k \in X$,

- $i \triangleright i=i$ for all $i \in X$.

The bijectivity of $\varphi_{i}$ can be expressed by the existence of a map $\triangleleft: X \times X \rightarrow X$ such that $(i \triangleright j) \triangleleft i=j=i \triangleright(j \triangleleft i)$ for all $i, j \in X$. Then

$$
k \triangleright(i \triangleleft j)=(k \triangleright i) \triangleleft(k \triangleright j), \quad(i \triangleleft j) \triangleleft k=(i \triangleleft k) \triangleleft(j \triangleleft k)
$$

for all $i, j, k \in X$. A crossed set is a quandle $X$ such that for all $i, j \in X, i \triangleright j=j$ implies $j \triangleright i=i$. Unions of conjugacy classes of a group with the binary operation of conjugation are examples of crossed sets.

Notation 1.4. In what follows, we use the following notation.

(1) To describe a finite quandle $X$ we may assume that $X=\{1, \ldots, n\}$ for some $n \in \mathbb{N}$ and then write $X: \varphi_{1} \varphi_{2} \cdots \varphi_{n}$ to denote the quandle structure on $X$ given by $\varphi_{1}, \ldots, \varphi_{n}$.

(2) Let $G$ be a group and $g \in G$. The quandle structure on the conjugacy class of $g$ in $G$ will be denoted by $g^{G}$.

The inner group of a quandle $X$ is the group $\operatorname{Inn}(X)=\left\langle\varphi_{i}: i \in X\right\rangle$. We say that a quandle $X$ is indecomposable if the inner group $\operatorname{Inn}(X)$ acts transitively on $X$. Also, $X$ is decomposable if it is not indecomposable.

Remark 1.5. Crossed sets of size at most three are well known. If $X$ is a crossed set and $1 \leq|X| \leq 2$, then $X$ is trivial (or commutative), that is, $i \triangleright j=j$ for all $i, j \in X$. If $|X|=3$ and $X$ is non-trivial, then $i \triangleright j=k$ for all pairwise different elements $i, j, k \in X$. Hence $X \simeq(12)^{\mathbb{S}_{3}}$. 
Remark 1.6. Using the classification of transitive groups of small degree, indecomposable quandles of small size were classified (up to isomorphism) in [24]. The list of indecomposable quandles of size $\leq 6$ is

$$
\begin{aligned}
& \{1\} \text { : id, } \\
& (12)^{\mathbb{S}_{3}}:(23)(13)(12) \text {, } \\
& (123)^{\mathbb{A}_{4}}: \text { (243) (134) (142) (123), } \\
& \operatorname{Aff}(5,2): \text { (2354) (1534) (1452) (1325) (1243), } \\
& \operatorname{Aff}(5,3): \text { (2453) (1435) (1254) (1523) (1342), } \\
& \operatorname{Aff}(5,4) \text { : (25)(34) (13)(45) (15)(24) (12)(35) (14)(23), } \\
& (12)^{\mathbb{S}_{4}}: \text { (23)(56) (13)(45) (12)(46) (25)(36) (16)(24) (15)(34), } \\
& (1234)^{\mathbb{S}_{4}}: \text { (2436) (1654) (1456) (1253) (2634) (1352). }
\end{aligned}
$$

Let $X$ be a quandle and let $G_{X}$ denote its enveloping group

$$
G_{X}=\left\langle x_{i}: i \in X\right\rangle /\left(x_{i} x_{j}=x_{i \triangleright j} x_{i} \text { for all } i, j \in X\right) .
$$

This group is $\mathbb{Z}$-graded with $\operatorname{deg}\left(x_{i}\right)=1$ for all $i \in X$.

Remark 1.7 (Universal property). For any group $G$ and any map $f: X \rightarrow G$ satisfying $f(x \triangleright y)=f(x) f(y) f(x)^{-1}$ there exists a unique group homomorphism $g: G_{X} \rightarrow G$ such that $f=g \circ \partial$, where $\partial: X \rightarrow G_{X}, i \mapsto x_{i}$, see for example [3, Lemma 1.6].

Let $X$ be a finite indecomposable quandle. By [11, Lemmas 2.17 and 2.18],

$$
x_{i}^{\left|\varphi_{i}\right|}=x_{j}^{\left|\varphi_{j}\right|}
$$

for all $i, j \in X$. This implies that the subgroup

$$
K=\left\langle x_{i}^{\left|\varphi_{i}\right|}: i \in X\right\rangle
$$

is cyclic and central. The finite enveloping group is the finite group $\overline{G_{X}}=G_{X} / K$, see [11, Lemma 2.19]. Let $\pi: G_{X} \rightarrow \overline{G_{X}}$ be the canonical surjection.

A quandle $X$ is said to be injective if the map $\partial: X \rightarrow G_{X}, i \mapsto x_{i}$, is injective. For example, the group $\overline{G_{X}}$ can be used to test indecomposable quandles for injectivity.

Lemma 1.8. Let $X$ be a finite indecomposable quandle and let $u \in G_{X}$. Then the following hold:

(1) The restriction of $\pi$ to the class $u^{G_{X}}$ is a quandle isomorphism.

(2) $X$ is injective if and only if $X \stackrel{\partial}{\rightarrow} G_{X} \stackrel{\pi}{\rightarrow} \overline{G_{X}}$ is injective. 
Proof. Let $v \in G_{X}$ and assume that $u$ and $v$ are conjugate. Then $u$ and $v$ have the same $\mathbb{Z}$-degree in $G_{X}$. Now, if $\pi u=\pi v$, then $u=v x_{1}^{m\left|\varphi_{1}\right|}$ for some $m \in \mathbb{Z}$. The $\mathbb{Z}$-graduation of $G_{X}$ implies that $m=0$ and hence $u=v$. Thus (1) is proved. Now (2) follows from (1).

Corollary 1.9. Let $X$ be a finite indecomposable quandle and

$$
M=\max \left\{|\mathcal{O}|: \mathcal{O} \text { is a conjugacy class of } \overline{G_{X}}\right\} .
$$

Then every conjugacy class of $G_{X}$ has at most $M$ elements.

Lemma 1.10. Let $X$ be a finite indecomposable quandle. Then $G_{X} \sim \overline{G_{X}}$.

Proof. Let $\pi: G_{X} \rightarrow \overline{G_{X}}$ be the canonical surjection. Since the elements of the group $\left[G_{X}, G_{X}\right]$ have degree zero,

$$
\operatorname{ker} \pi \cap\left[G_{X}, G_{X}\right]=\left\langle x_{1}^{\left|\varphi_{1}\right|}\right\rangle \cap\left[G_{X}, G_{X}\right]=1 .
$$

Then the claim follows from Lemma $1.2(1)$ with $G=G_{X}, K=\operatorname{ker} \pi$.

We conclude the subsection on quandles with two technical lemmas needed for the proof of our main result.

Lemma 1.11. Let $X$ be a crossed set, $Y \subseteq X$ be a subset, and

$$
C(Y)=\{i \in X: i \triangleright j=j \text { for all } j \in Y\} \text {. }
$$

Assume that $Y \cup C(Y)=X$. Then $X \triangleright Y=Y$.

Proof. Let $p, q \in C(Y)$ and $i \in Y$. Then

$$
(p \triangleright q) \triangleright i=p \triangleright(q \triangleright i)=p \triangleright i=i
$$

and hence

$$
C(Y) \triangleright C(Y) \subseteq C(Y) .
$$

Since $Y \triangleright C(Y)=C(Y)$ by the definition of $C(Y)$ and since $X=Y \cup C(Y)$, we conclude that

$$
X \triangleright C(Y) \subseteq C(Y) .
$$

Since $i \triangleleft p=i \triangleleft q=i$, we obtain similarly that

$$
C(Y) \triangleleft C(Y) \subseteq C(Y) \text { and } C(Y) \triangleleft X \subseteq C(Y) .
$$

Hence $X \triangleright C(Y)=C(Y)$ and $X \triangleright(X \backslash C(Y))=X \backslash C(Y)$.

Let $k \in Y \cap C(Y)$. Then we have $i \triangleright k=k$ for all $i \in Y$ since $k \in C(Y)$, and $i \triangleright k=k$ for all $i \in C(Y)$ since $k \in Y$. Since $Y \cup C(Y)=X$, we conclude that $X \triangleright\{k\}=\{k\}$ for all $k \in Y \cap C(Y)$. This and the first paragraph imply that $X \triangleright Y=Y$. 
Lemma 1.12. Let $X=Y_{1} \cup Y_{2}$ be a finite quandle, where $Y_{1}$ and $Y_{2}$ are disjoint $\operatorname{Inn}(X)$-orbits. Assume that there exists an isomorphism of quandles $g: Y_{1} \rightarrow Y_{2}$ and that $Y_{1}$ is commutative. Then the quandle $X$ is isomorphic to the quandle structure on $\{1, \ldots, 2 n\}$ given by

$$
\varphi_{i}= \begin{cases}(n+1 \cdots 2 n) & \text { if } 1 \leq i \leq n, \\ (1 \cdots n) & \text { if } n+1 \leq i \leq 2 n .\end{cases}
$$

Proof. Without loss of generality we may assume that $Y_{1}=\{1, \ldots, n\}$ and that $Y_{2}=\{n+1, \ldots, 2 n\}$. For all $i \in Y_{1}$ and $j \in Y_{2}$ the permutations $\varphi_{i}$ and $\varphi_{j}$ commute, since $\operatorname{supp} \varphi_{i} \subseteq Y_{2}$ and $\operatorname{supp} \varphi_{j} \subseteq Y_{1}$. Further,

$$
\varphi_{j \triangleright i}=\varphi_{j} \varphi_{i} \varphi_{j}^{-1}=\varphi_{i}
$$

As $\varphi_{k \triangleright i}=\varphi_{i}$ for all $k \in Y_{1}$ by the commutativity of $Y_{1}$, we conclude that $\varphi_{i}=\varphi_{l}$ for all $i, l \in Y_{1}$ since $Y_{1}$ is an $\operatorname{Inn}(X)$-orbit.

Since $Y_{1}$ and $Y_{2}$ are isomorphic, we have $\varphi_{i}=\varphi_{l}$ for all $i, l \in Y_{2}$. Since $Y_{1}$ is an $\operatorname{Inn}(X)$-orbit, it is a $\varphi_{n+1}$-orbit and hence for all $j \in Y_{2}$ the permutation $\varphi_{j}$ is a cycle of length $\left|Y_{1}\right|$. This implies the claim.

\section{Groups with finite-dimensional Nichols algebras}

Here we introduce the groups that realize the examples of decomposable quandles which are essential for our classification. These quandles are

$$
\begin{aligned}
& Z_{T}^{4,1}:(243)(134)(142)(123) \mathrm{id}, \\
& Z_{2}^{2,2}:(24)(13)(24)(13) \\
& Z_{3}^{3,1}:(23)(13)(12) \mathrm{id} \\
& Z_{3}^{3,2}:(23)(45)(13)(45)(12)(45)(123)(132) \\
& Z_{4}^{4,2}:(24)(56)(13)(56)(24)(56)(13)(56)(1234)(1432)
\end{aligned}
$$

First we study the dimension of group representations.

Lemma 2.1. Let $G$ be a group, $x \in G$, and $d \in \mathbb{N}$. Suppose that $\left[G: G^{x}\right]$ is finite. If $\operatorname{dim}_{\mathbb{K}} V \leq d$ for any finite-dimensional absolutely simple $\mathbb{K} G^{x}$-module $V$, then

$$
\operatorname{dim}_{\mathbb{K}} U \leq d\left[G: G^{x}\right]
$$

for any finite-dimensional absolutely simple $\mathbb{K} G$-module $U$. In particular,

$$
\operatorname{dim}_{\mathbb{K}} U \leq\left[G: G^{x}\right]
$$

if $G^{x}$ is abelian. 
Proof. We may assume that the field $\mathbb{K}$ is algebraically closed. Let $U$ be a simple $\mathbb{K} G$-module with $\operatorname{dim}_{\mathbb{K}} U<\infty$ and let $V$ be a simple $\mathbb{K} G^{x}$-submodule of $U$. Then $U=\mathbb{K} G V$ is an epimorphic image of $\mathbb{K} G \otimes_{\mathbb{K} G^{x}} V$ and $\operatorname{dim}_{\mathbb{K}} V \leq d$, and hence $\operatorname{dim}_{\mathbb{K}} U \leq d\left[G: G^{x}\right]$. Now the second claim follows, as finite-dimensional absolutely simple modules of abelian groups are one-dimensional.

\subsection{The group $T$}

Let us consider the group

$$
T=\langle z\rangle \times\left\langle x_{1}, x_{2}, x_{3}, x_{4}: x_{i} x_{j}=x_{\varphi_{i}(j)} x_{i}, i, j \in\{1,2,3,4\}\right\rangle,
$$

where $\left\{\varphi_{i}: 1 \leq i \leq 4\right\}$ is the set of permutations that defines $(123)^{\mathbb{A}_{4}}$. This group is not nearly abelian, see [15, Definition 3.1], since the commutator subgroup $[T, T]$ is not cyclic. For example $\left[x_{1}, x_{2}\right]$ and $\left[x_{1}, x_{3}\right]$ do not commute. (One can prove that $[T, T] \simeq Q_{8}$, the quaternion group of eight elements.)

Example 2.2. Let $Z_{T}^{4,1}=x_{1}^{T} \cup z^{T}$, see Notation 1.4(2). Then the group $T$ is isomorphic to the enveloping group of $Z_{T}^{4,1}$.

Lemma 2.3. Let $G$ be an epimorphic image of $T$. Then the following hold:

(1) $G$ has abelian centralizers.

(2) Every conjugacy class of $G$ has at most six elements.

(3) Every finite-dimensional absolutely simple $\mathbb{K} G$-module has dimension at most four.

Proof. By Lemma 1.3, we may replace $G$ by a group which is isoclinic to $G$. Let $K \triangleleft T$ with $G=T / K$. By Lemma 1.2(1) we may assume that $K \subseteq[T, T]$. Let $X=(123)^{\mathbb{A}_{4}}$. Since $T \sim G_{X}$ and $G_{X} \sim \overline{G_{X}}$ by Lemma $1.10, T / K \sim \overline{G_{X}} / L$ for some $L \triangleleft \overline{G_{X}}$ by Lemma 1.2 (2). Now $\overline{G_{X}} \simeq \mathbf{S L}(2,3)$ and the only non-trivial normal subgroups of $\mathbf{S L}(2,3)$ are its commutator subgroup and its center. Since all quotients of $\mathbf{S L}(2,3)$ have abelian centralizers, claim (1) holds.

To prove (2) we use Corollary 1.9 , as every conjugacy class of $\mathbf{S L}(2,3)$ has at most six elements. Then Lemma 2.1 and (1) and $|X|=4$ imply (3).

\subsection{The groups $\Gamma_{n}$}

Let $n \in \mathbb{N}_{\geq 2}$. Recall from [15] that

$$
\Gamma_{n}=\left\langle g, h, \epsilon: h g=\epsilon g h, g \epsilon=\epsilon^{-1} g, h \epsilon=\epsilon h, \epsilon^{n}=1\right\rangle .
$$

(These groups were denoted by $G_{n}$ in [15].) Any element of $\Gamma_{n}$ can be written uniquely as $\epsilon^{i} h^{j} g^{k}$, where $0 \leq i \leq n-1$ and $j, k \in \mathbb{Z}$. By [15, Section 3], the 
conjugacy classes of $\Gamma_{n}$ are

$$
\begin{aligned}
z^{\Gamma_{n}} & =\{z\}, & (g z)^{\Gamma_{n}} & =\left\{\epsilon^{m} g z: 0 \leq m \leq n-1\right\}, \\
\left(h^{j} z\right)^{\Gamma_{n}} & =\left\{h^{j} z, \epsilon^{-j} h^{j} z\right\}, & (h g z)^{\Gamma_{n}} & =\left\{\epsilon^{m} h g z: 0 \leq m \leq n-1\right\},
\end{aligned}
$$

where $z \in Z\left(\Gamma_{n}\right)=\left\langle\epsilon^{-1} h^{2}, h^{n}, g^{2}\right\rangle$ and $1 \leq j \leq \frac{n}{2}$. The centralizers

$$
\left(\Gamma_{n}\right)^{g z}=\left\langle\epsilon^{-1} h^{2}, g, h^{n}\right\rangle, \quad\left(\Gamma_{n}\right)^{h g z}=\left\langle\epsilon^{-1} h^{2}, h g, h^{n}\right\rangle, \quad\left(\Gamma_{n}\right)^{h^{j} z}=\left\langle\epsilon, h, g^{2}\right\rangle
$$

are abelian. The commutator subgroup is $\left[\Gamma_{n}, \Gamma_{n}\right]=\langle\epsilon\rangle$.

Now we show four examples of decomposable quandles.

Example 2.4. Let $Z_{2}^{2,2}=h^{\Gamma_{2}} \cup g^{\Gamma_{2}}$. Then $Z_{2}^{2,2} \simeq \mathbb{D}_{4}$, the dihedral quandle of four elements. The enveloping group of $Z_{2}^{2,2}$ is

$$
\left\langle x_{1}, x_{2}, x_{3}, x_{4}: x_{i} x_{j}=x_{2 i-j(\bmod 4)} x_{i}, i, j \in\{1,2,3,4\}\right\} \simeq \Gamma_{2} .
$$

The isomorphism is given by $x_{1} \mapsto g, x_{2} \mapsto h$.

Example 2.5. Let $Z_{3}^{3,1}=g^{\Gamma_{3}} \cup\{\epsilon h\}$. Note that $\epsilon h \in Z\left(\Gamma_{3}\right)$. The enveloping group of $Z_{3}^{3,1}$ is isomorphic to

$$
\langle z\rangle \times\left\langle x_{1}, x_{2}, x_{3}: x_{i} x_{j}=x_{2 i-j(\bmod 3)} x_{i}, i, j \in\{1,2,3\}\right\} \simeq \Gamma_{3} .
$$

The latter isomorphism is given by $z \mapsto \epsilon h, x_{1} \mapsto g, x_{2} \mapsto \epsilon g$ and $x_{3} \mapsto \epsilon^{2} g$.

Example 2.6. Let $Z_{3}^{3,2}=g^{\Gamma_{3}} \cup h^{\Gamma_{3}}$. The enveloping group of $Z_{3}^{3,2}$ is isomorphic to $\Gamma_{3}$.

Example 2.7. Let $Z_{4}^{4,2}=g^{\Gamma_{4}} \cup h^{\Gamma_{4}}$. The enveloping group of $Z_{4}^{4,2}$ is isomorphic to $\Gamma_{4}$.

Lemma 2.8. Let $G$ be an epimorphic image of $\Gamma_{n}$ for some $n \geq 2$. Then the following hold:

(1) G has abelian centralizers.

(2) Every conjugacy class of $G$ has at most $n$ elements.

(3) Every finite-dimensional absolutely simple $\mathbb{K} G$-module has dimension at most two.

Proof. Let $p: \Gamma_{n} \rightarrow G$ be the canonical map. If $\epsilon^{k} \in \operatorname{ker} p$ for some $k>0$, then $G$ is also an epimorphic image of $\Gamma_{k}$. Since $\left[\Gamma_{n}, \Gamma_{n}\right]=\langle\epsilon\rangle$, we may assume that ker $p \cap\left[\Gamma_{n}, \Gamma_{n}\right]=1$. Hence $\Gamma_{n} \sim G$ by Lemma 1.2(1). Therefore claim (1) follows from Lemma 1.3 , since $\Gamma_{n}$ has abelian centralizers.

Claim (2) follows from the description of conjugacy classes of $\Gamma_{n}$. Finally (3) follows from Lemma 2.1 since $h^{G}$ has two elements. 


\section{Weyl groupoids of rank two}

Let us consider the map

$$
\eta: \mathbb{Z} \rightarrow \mathbf{S L}(2, \mathbb{Z}), \quad \eta(c)=\left(\begin{array}{cc}
c & -1 \\
1 & 0
\end{array}\right) .
$$

A finite sequence $\left(c_{1}, c_{2}, \ldots, c_{n}\right), n \in \mathbb{N}$, of positive integers is a characteristic sequence if $\eta\left(c_{1}\right) \cdots \eta\left(c_{n}\right)=-\mathrm{id}$, and the entries of the first column of the matrix $\eta\left(c_{1}\right) \cdots \eta\left(c_{i}\right)$ are non-negative integers for all $i<n$. We denote by $\mathcal{A}^{+}$ the set of characteristic sequences. By [10, Lemma 5.2],

$$
\begin{aligned}
\left(c_{1}, c_{2}, \ldots, c_{n}\right) \in \mathcal{A}^{+}, c_{2}=1, n \geq 4 & \\
& \Longleftrightarrow\left(c_{1}-1, c_{3}-1, c_{4}, \ldots, c_{n}\right) \in \mathcal{A}^{+} .
\end{aligned}
$$

Lemma 3.1. Let $\left(c_{1}, c_{2}, \ldots, c_{n}\right) \in \mathcal{A}^{+}$. Then $n \geq 3$ and there exists an index $i$ with $i \in\{1, \ldots, n\}$ such that

- $c_{i}=1$ and $c_{i+1} \in\{1,2,3\}$,

or

- $c_{i}=1$ and $c_{i-1} \in\{1,2,3\}$,

where $c_{0}=c_{n}$ and $c_{n+1}=c_{1}$.

Proof. Let $\left(c_{1}, c_{2}, \ldots, c_{n}\right) \in \mathcal{A}^{+}$. If $n \leq 3$, then we have $\left(c_{1}, \ldots, c_{n}\right)=(1,1,1)$ by [10, Proposition 5.3(4)]. Hence we may assume that $n>3$. By [10, Corollary 4.2], there exists an $i \in\{1, \ldots, n\}$ such that $c_{i}=1$. Further,

$$
\left(c_{j}, c_{j+1}, \ldots, c_{n}, c_{1}, \ldots, c_{j-1}\right) \in \mathcal{A}^{+} \quad \text { for all } j \in\{1, \ldots, n\}
$$

by [10, Proposition 5.3(2)]. Also, $c_{i}=1$ implies that $c_{i-1}, c_{i+1}>1$ by (3.1). Therefore, without loss of generality we may assume that $c=\left(b_{1}, b_{2}, \ldots, b_{r}\right)$, where $b_{i}=\left(c_{i 1}, 1, c_{i 2}, 1, \ldots, c_{i m_{i}}, 1, c_{i m_{i}+1}\right)$ and $c_{i j} \geq 2$ for all $1 \leq i \leq r$ and $1 \leq j \leq m_{i}+1$, or $c=\left(d_{1}, 1, \ldots, d_{m}, 1\right)$ with $n=2 m, d_{1}, \ldots, d_{m} \geq 2$.

Assume first that $c=\left(b_{1}, b_{2}, \ldots, b_{r}\right)$. Then $m_{i} \geq 2$ for at least one $i$. By applying (3.1) several times we obtain that $\left(b_{1}^{\prime}, b_{2}^{\prime}, \ldots, b_{r}^{\prime}\right) \in \mathcal{A}^{+}$, where

$$
b_{i}^{\prime}=\left(c_{i 1}-1, c_{i 2}-2, c_{i 3}-2, \ldots, c_{i m_{i}}-2, c_{i m_{i}+1}-1\right) \quad \text { for all } 1 \leq i \leq r .
$$

Since $\left(b_{1}^{\prime}, b_{2}^{\prime}, \ldots, b_{r}^{\prime}\right) \in \mathcal{A}^{+}$, by [10, Corollary 4.2] there exists an $i \in\{1, \ldots, r\}$ such that $c_{i 1}-1=1$ or $c_{i m_{i}+1}-1=1$ or $c_{i j}-2=1$ for some $j \in\left\{2, \ldots, m_{i}\right\}$. Then the lemma holds. 
Now assume that $c=\left(d_{1}, 1, d_{2}, 1, \ldots, d_{m}, 1\right), n=2 m$. If $d_{i}=2$ for some $1 \leq i \leq m$, then we are done. Otherwise, after applying (3.1) $m$ times we obtain that $\left(d_{1}-2, d_{2}-2, \ldots, d_{m}-2\right) \in \mathcal{A}^{+}$. Hence there exists an $i \in\{1, \ldots, m\}$ such that $d_{i}-2=1$. This implies the lemma.

Cartan schemes of rank two, their Weyl groupoids and their root systems were studied in [10]. An indecomposable Cartan matrix $C \in \mathbb{Z}^{2 \times 2}$ of finite type is a matrix of the form $\left(\begin{array}{cc}2 & -c_{1} \\ -c_{2} & 2\end{array}\right)$, where $c_{1}, c_{2} \in \mathbb{N}, 1 \leq c_{1} c_{2} \leq 3$.

Corollary 3.2. Let $\mathcal{C}=\mathcal{C}\left(\{1,2\}, A,\left(\rho_{i}\right)_{i \in\{1,2\}},\left(C^{a}\right)_{a \in A}\right)$ be a connected Cartan scheme admitting a finite irreducible root system $\left(R^{a}\right)_{a \in A}$. Then there exists an element $a \in A$ such that $C^{a} \in \mathbb{Z}^{2 \times 2}$ is an indecomposable Cartan matrix of finite type.

Proof. Let $a \in A, n=\left|R_{+}^{a}\right|, a_{1}, \ldots, a_{2 n} \in A, c_{1}, \ldots, c_{2 n} \in \mathbb{N}$ such that

$$
\begin{aligned}
& a_{2 r-1}=\left(\rho_{2} \rho_{1}\right)^{r-1}(a), \quad a_{2 r}=\rho_{1}\left(\rho_{2} \rho_{1}\right)^{r-1}(a) \text {, } \\
& c_{2 r-1}=-c_{12}^{a_{2 r-1}}, \quad c_{2 r}=-c_{21}^{a_{2 r}}
\end{aligned}
$$

for all $r \in\{1,2, \ldots, n\}$. Then $\left(c_{1}, \ldots, c_{n}\right) \in \mathcal{A}^{+}$by [10, Proposition 6.5]. By Lemma 3.1, there exists an $i$ such that $c_{i}=1$ and $c_{i+1} \in\{1,2,3\}$, or $c_{i}=1$ and $c_{i-1} \in\{1,2,3\}$. This implies the corollary.

\section{Nichols algebras over groups}

Recall that a Yetter-Drinfeld module over a group $G$ is a $\mathbb{K} G$-module

$$
V=\bigoplus_{g \in G} V_{g}
$$

such that $h V_{g} \subseteq V_{h g h^{-1}}$ for all $g, h \in G$.

Lemma 4.1. Let $G$ be an epimorphic image of one of the groups $\Gamma_{2}, \Gamma_{3}, \Gamma_{4}$ or $T$. Then every finite-dimensional absolutely simple Yetter-Drinfeld module over $G$ has dimension at most six.

Proof. Any simple Yetter-Drinfeld module over $G$ is uniquely given by a conjugacy class $\mathcal{O}$ of $G$ and an irreducible representation $\rho$ of the centralizer of an element of $\mathcal{O}$. In this case, $\operatorname{dim} V=|\mathcal{O}| \operatorname{deg} \rho$. Hence the claim follows from Lemmas 2.8 and 2.3 .

For the study of Nichols algebras over groups the Weyl groupoid of a tuple of simple Yetter-Drinfeld modules plays an important role. For the definition we refer to [15]. 
Theorem 4.2. Let $\theta \in \mathbb{N}$, let $H$ be a Hopf algebra with bijective antipode and let $M=\left(M_{1}, \ldots, M_{\theta}\right)$, where each $M_{i}$ is a simple Yetter-Drinfeld module over $H$. Assume that $M$ admits all reflections and that the Weyl groupoid $W(M)$ is finite. Then $\mathcal{B}(M)$ is decomposable and admits a finite root system of type $\mathcal{C}(M)$.

Proof. By [15, Corollary 2.4], $\mathscr{B}(M)$ is decomposable. Then the theorem becomes precisely [15, Theorem 2.3].

For any Yetter-Drinfeld module $V$ over a Hopf algebra $H$ with bijective antipode let $[V]$ denote the isomorphism class of $V$. The first step in the proof of Theorem 4.5 will be the following claim.

Proposition 4.3. Let $H$ be a Hopf algebra with bijective antipode. Further, let $M=\left(M_{1}, M_{2}\right)$ be a pair of simple Yetter-Drinfeld modules over $H$. Assume that $M$ admits all reflections and $\mathcal{W}(M)$ is finite. If (id $\left.-c_{M_{2}, M_{1}} c_{M_{1}, M_{2}}\right) \neq 0$, then there exists a pair $N=\left(N_{1}, N_{2}\right)$ of simple Yetter-Drinfeld modules over $H$, such that $[N]=\left(\left[N_{1}\right],\left[N_{2}\right]\right) \in \mathcal{W}(M)$ and $1 \leq a_{12}^{[N]} a_{21}^{[N]} \leq 3$.

Proof. Since the pair $M$ admits all reflections and (id $\left.-c_{M_{2}, M_{1}} c_{M_{1}, M_{2}}\right) \neq 0$, the set of real roots of $W(M)$ is irreducible. Therefore the proposition follows from Corollary 3.2.

Theorem 4.4. Let $\mathbb{K}$ be a field, $G$ be a non-abelian group, and $V$ and $W$ be two Yetter-Drinfeld modules over $G$. Assume that $G$ is generated as a group by $\operatorname{supp}(V \oplus W)$, supp $V$ and $\operatorname{supp} W$ are conjugacy classes of $G,(\operatorname{ad} V)^{2}(W)=0$ and $(\operatorname{ad} W)^{4}(V)=0$. If $\left(\mathrm{id}-c_{W, V} c_{V, W}\right)(V \otimes W) \neq 0$, then $\operatorname{supp}(V \oplus W)$ is isomorphic to one of the quandles

$$
Z_{T}^{4,1}, Z_{2}^{2,2}, Z_{3}^{3,1}, Z_{3}^{3,2} \text { and } Z_{4}^{4,2},
$$

and $G$ is isomorphic to an epimorphic image of the corresponding enveloping groups $T, \Gamma_{2}, \Gamma_{3}, \Gamma_{3}$ and $\Gamma_{4}$, respectively.

Before proving Theorem 4.4 we turn our attention to some consequences.

Theorem 4.5. Let $\mathbb{K}$ be a field, let $G$ be a non-abelian group, and let $V$ and $W$ be finite-dimensional absolutely simple Yetter-Drinfeld modules over G. Assume that $G$ is generated by $\operatorname{supp}(V \oplus W)$, the pair $(V, W)$ admits all reflections, and the Weyl groupoid of $(V, W)$ is finite. If (id $\left.-c_{W, V} c_{V, W}\right)(V \otimes W) \neq 0$, then $G$ is isomorphic to an epimorphic image of $\Gamma_{n}$ for some $n \in\{2,3,4\}$ or T. Moreover, $\operatorname{dim} V \leq 6$ and $\operatorname{dim} W \leq 6$. 
Proof. Proposition 4.3 implies that after changing the object of $\mathcal{W}(V, W)$, and possibly interchanging the roles of $V$ and $W$, we may assume that $(\operatorname{ad} V)(W) \neq 0$, $(\operatorname{ad} V)^{2}(W)=0$ and $(\operatorname{ad} W)^{4}(V)=0$. Theorem 4.4 implies that the group $G$ is an epimorphic image of $\Gamma_{n}$ for $n \in\{2,3,4\}$ or an epimorphic image of $T$. After applying reflections to the pair $(V, W)$ we obtain new pairs $\left(V^{\prime}, W^{\prime}\right)$ of absolutely simple Yetter-Drinfeld modules over $G$. Thus the claim follows from Lemma 4.1.

Corollary 4.6. Let $\mathbb{K}$ be a field, let $G$ be a non-abelian group, and let $V$ and $W$ be finite-dimensional absolutely simple Yetter-Drinfeld modules over $G$. Assume that $G$ is generated by $\operatorname{supp}(V \oplus W)$ and that $\mathscr{B}(V \oplus W)$ is finite-dimensional. If $\left(\mathrm{id}-c_{W, V} c_{V, W}\right)(V \otimes W) \neq 0$, then $\operatorname{dim} V \leq 6$ and $\operatorname{dim} W \leq 6$.

Proof. Assume that $\mathcal{B}(V \oplus W)$ is finite-dimensional. Then $(V, W)$ admits all reflections by [4, Corollary 3.18] and the Weyl groupoid is finite by [4, Proposition 3.23]. So Theorem 4.5 applies.

\section{Proof of Theorem 4.4}

The key of our proof is Proposition 5.5 which allows us to construct non-zero elements of $(\operatorname{ad} V)^{m}(W)$ for any two Yetter-Drinfeld modules $V$ and $W$ over a group $G$ and for any $m \in \mathbb{N}$ under some assumption on $G$. Then we split our analysis into two parts depending on the question whether supp $V$ and $\operatorname{supp} W$ commute. Finally, we prove Theorem 4.4 in Section 5.4.

In the whole section, let $G$ be a non-abelian group and let $V=\bigoplus_{s \in G} V_{s}$ and $W=\bigoplus_{t \in G} W_{t}$ be Yetter-Drinfeld modules over $G$.

\subsection{General considerations}

Lemma 5.1. Let $G$ be a group, and $g, h \in G$. Assume that $G$ is generated by $g^{G}$ and $h^{G}$. Then $G=A B$, where $A=\left\langle g^{G}\right\rangle, B=\left\langle h^{G}\right\rangle$, and

$$
A B=\{a b: a \in A, b \in B\} .
$$

Proof. Let $r \in g^{G}$ and $s \in h^{G}$. Writing $s r=r\left(r^{-1} s r\right)$ we conclude that

$$
g^{G} h^{G}=h^{G} g^{G} .
$$

From this the claim follows.

Recall that $S_{n} \in \operatorname{End}\left(V^{\otimes n}\right)$, where $n \in \mathbb{N}$, denotes the quantum symmetrizer. 
Lemma 5.2 ([16, Proposition 6.5]). Let $n \in \mathbb{N}$. Then

$$
(\operatorname{ad} V)^{n}(W) \simeq\left(S_{n} \otimes \mathrm{id}\right) T_{n}\left(V^{\otimes n} \otimes W\right),
$$

where $T_{n} \in \operatorname{End}\left(V^{\otimes n} \otimes W\right)$ is defined by

$$
T_{n}=\left(\mathrm{id}-c_{n, n+1}^{2} c_{n-1, n} \cdots c_{1,2}\right) \cdots\left(\mathrm{id}-c_{n, n+1}^{2} c_{n-1, n}\right)\left(\mathrm{id}-c_{n, n+1}^{2}\right) .
$$

Lemma 5.3 ([15, Theorem 1.1]). Let $\varphi_{0}=0, X_{0}^{V, W}=W$, and

$$
\begin{aligned}
\varphi_{m} & =\mathrm{id}-c_{V} \otimes(m-1) \otimes W, V \\
X_{m}^{V, W} & =\varphi_{m}\left(V \otimes X_{m-1} \otimes(m-1) \otimes W\right.
\end{aligned}
$$

for all $m \geq 1$. Then

$$
\left(S_{n+1} \otimes \mathrm{id}_{W}\right) T_{n+1}=\varphi_{n+1}\left(\mathrm{id}_{V} \otimes S_{n} \otimes \mathrm{id}_{W}\right)\left(\mathrm{id}_{V} \otimes T_{n}\right)
$$

and $(\operatorname{ad} V)^{n}(W) \simeq X_{n}^{V, W}$ for all $n \in \mathbb{N}_{0}$.

Let $m \in \mathbb{N}_{0}$. Recall that an element of $V^{\otimes m} \otimes W$ has degree $\left(r_{1}, \ldots, r_{m}, s\right)$, where $r_{1}, \ldots, r_{m}, s \in G$, if it is contained in $V_{r_{1}} \otimes \cdots V_{r_{m}} \otimes W_{s}$.

Let $r_{1}, r_{2}, \ldots, r_{m} \in \operatorname{supp} V$ and $s \in \operatorname{supp} W$, and write

$$
Q_{m}\left(r_{1}, \ldots, r_{m}, s\right)=\left(S_{m} \otimes \mathrm{id}\right) T_{m}\left(V_{r_{1}} \otimes \cdots \otimes V_{r_{m}} \otimes W_{s}\right) \subseteq V^{\otimes m} \otimes W .
$$

Although the vector space $V^{\otimes m} \otimes W$ is graded by $(\operatorname{supp} V)^{m} \times \operatorname{supp} W$, the subspace $Q_{m}\left(r_{1}, \ldots, r_{m}, s\right)$ is usually not graded. For $t \in V^{\otimes m} \otimes W$ we write supp $t$ for the set of $d \in(\operatorname{supp} V)^{m} \times \operatorname{supp} W$, such that the homogeneous component of $t$ of degree $d$ is non-zero. We let

$$
\operatorname{supp} Q=\{\operatorname{supp} t: t \in Q\}
$$

for all subspaces $Q \subseteq V^{\otimes m} \otimes W$.

Remark 5.4. Let $m \in \mathbb{N}$. By Lemma 5.2, we have $(\operatorname{ad} V)^{m}(W)=0$ if and only if $\operatorname{supp} Q_{m}\left(r_{1}, \ldots, r_{m}, s\right)=0$ for all $r_{1}, \ldots, r_{m} \in \operatorname{supp} V, s \in \operatorname{supp} W$.

Proposition 5.5. Let $m \in \mathbb{N}_{0}$. Further, let $p_{1}, \ldots, p_{m}, r_{1}, \ldots, r_{m} \in \operatorname{supp} V$ and $p_{m+1}, s \in \operatorname{supp} W$ such that

$$
\left(p_{1}, \ldots, p_{m}, p_{m+1}\right) \in \operatorname{supp} Q_{m}\left(r_{1}, \ldots, r_{m}, s\right) .
$$

Let $p \in \operatorname{supp} V$ and $i \in\{1, \ldots, m+1\}$ and assume that

$$
\begin{gathered}
p_{i} \triangleright p \neq p, \quad p_{j} \triangleright p=p \text { for all } j \text { with } i<j \leq m+1, \\
p \notin\left\{p_{j}: 1 \leq j \leq m\right\} \cup\left\{\left(p_{j+1} p_{j+2} \cdots p_{m+1}\right)^{-1} \triangleright p_{j}: 1 \leq j<i\right\} .
\end{gathered}
$$

Then $\left(p \triangleright p_{1}, \ldots, p \triangleright p_{i-1}, p, p_{i}, \ldots, p_{m}, p_{m+1}\right) \in \operatorname{supp} Q_{m+1}\left(p, r_{1}, \ldots, r_{m}, s\right)$. 
Proof. Let $t \in Q_{m}\left(r_{1}, \ldots, r_{m}, s\right)$ and let $p \in \operatorname{supp} V$. By Lemma 5.3, we have $\varphi_{m+1}(v \otimes t) \in Q_{m+1}\left(p, r_{1}, \ldots, r_{m}, s\right)$ for all $v \in V_{p}$. Moreover, $\varphi_{m+1}(v \otimes t)$ is a sum of non-zero homogeneous tensors of degrees

$$
\begin{gathered}
\left(p \triangleright p_{1}^{\prime}, \ldots, p \triangleright p_{j-1}^{\prime}, p, p_{j}^{\prime}, \ldots, p_{m}^{\prime}, p_{m+1}^{\prime}\right), \\
\left(p \triangleright p_{1}^{\prime}, \ldots, p \triangleright p_{j-1}^{\prime}, p p_{j}^{\prime} \cdots p_{m+1}^{\prime} \triangleright p, p \triangleright p_{j}^{\prime}, \ldots, p \triangleright p_{m}^{\prime}, p \triangleright p_{m+1}^{\prime}\right)
\end{gathered}
$$

with $1 \leq j \leq m+1$, where $\left(p_{1}^{\prime}, \ldots, p_{m}^{\prime}, p_{m+1}^{\prime}\right) \in \operatorname{supp} t$. By the assumption on $p_{1}, \ldots, p_{m}, p_{m+1}$, the tuple

$$
\left(p \triangleright p_{1}, \ldots, p \triangleright p_{i-1}, p, p_{i}, p_{i+1}, \ldots, p_{m}, p_{m+1}\right)
$$

appears among the degrees in (5.3). It suffices to show that it appears exactly once. We split the proof into several cases.

Assume first that (5.4) is equal to ( $\left.p \triangleright p_{1}^{\prime}, \ldots, p \triangleright p_{j-1}^{\prime}, p, p_{j}^{\prime}, \ldots, p_{m}^{\prime}, p_{m+1}^{\prime}\right)$ for some $j \in\{1, \ldots, m+1\}$. There are three cases to consider. First, if $j<i$, then $p \triangleright p_{j}=p$ and hence $p=p_{j}$, a contradiction to (5.2). If $j=i$, then we obtain $p_{l}=p_{l}^{\prime}$ for all $l \in\{1, \ldots, m+1\}$ which gives us just the tuple we are looking at. Finally, if $j>i$, then $p=p_{j-1}$, again a contradiction to (5.2).

Now assume that (5.4) is equal to

$$
\left(p \triangleright p_{1}^{\prime}, \ldots, p \triangleright p_{j-1}^{\prime}, p p_{j}^{\prime} \cdots p_{m+1}^{\prime} \triangleright p, p \triangleright p_{j}^{\prime}, \ldots, p \triangleright p_{m}^{\prime}, p \triangleright p_{m+1}^{\prime}\right)
$$

for some $j \in\{1, \ldots, m+1\}$. Again there are three cases to consider.

If $j>i$, then

$$
p \triangleright p_{k}^{\prime}=p_{k} \text { for all } k \in\{j, j+1, \ldots, m+1\}, \quad p p_{j}^{\prime} \cdots p_{m+1}^{\prime} \triangleright p=p_{j-1} .
$$

By (5.1) we conclude that $p_{k}^{\prime}=p_{k}$ for all $k \in\{j, j+1, \ldots, m+1\}$ and $p=p_{j-1}$.

If $j=i$, then

$$
p \triangleright p_{k}^{\prime}=p_{k} \text { for all } k \in\{i, i+1, \ldots, m+1\}, \quad p p_{i}^{\prime} \cdots p_{m+1}^{\prime} \triangleright p=p .
$$

We conclude from (5.1) that $p_{k}^{\prime}=p_{k}$ for all $k \in\{i+1, \ldots, m+1\}, p \triangleright p_{i}^{\prime}=p_{i}$ and $p p_{i}^{\prime} \triangleright p=p$. This implies that

$$
p_{i} \triangleright p=\left(p \triangleright p_{i}^{\prime}\right) \triangleright p=p p_{i}^{\prime} p^{-1} \triangleright p=p p_{i}^{\prime} \triangleright p=p,
$$

a contradiction to (5.1).

Finally, assume that $1 \leq j<i$. Then $p p_{j}^{\prime} \cdots p_{m+1}^{\prime} \triangleright p=p \triangleright p_{j}$, or

$$
\left(p \triangleright p_{j}^{\prime}\right)\left(p \triangleright p_{j+1}^{\prime}\right) \cdots\left(p \triangleright p_{m+1}^{\prime}\right) \triangleright p=p \triangleright p_{j} .
$$

We conclude from this and the equality of (5.4) and (5.5) that

$$
\left(p \triangleright p_{j+1}\right) \cdots\left(p \triangleright p_{i-1}\right) p p_{i} \cdots p_{m+1} \triangleright p=p \triangleright p_{j} .
$$


The latter is equivalent to

$$
p p_{j+1} \cdots p_{i-1} p_{i} \cdots p_{m+1} \triangleright p=p \triangleright p_{j}
$$

which after cancelling $p \triangleright$ gives a contradiction to (5.2).

Remark 5.6. If $m=0$, then Proposition 5.5 reads as follows. Let $s \in \operatorname{supp} W$ and $p \in \operatorname{supp} V$ and assume that $s \triangleright p \neq p$. Then $(p, s) \in \operatorname{supp} Q_{1}(p, s)$.

Corollary 5.7. Let $m \geq 1$. Assume that the following hold:

(1) the quandle supp $V$ is indecomposable or supp $V=Y_{1} \cup Y_{2}$ is a decomposition into $\operatorname{Inn}(\operatorname{supp} V)$-orbits, and $x \triangleright Y_{1}=Y_{2}, x \triangleright Y_{2}=Y_{1}$ for all $x \in \operatorname{supp} W$.

(2) $(\operatorname{ad} V)^{m}(W) \neq 0,(\operatorname{ad} V)^{m+1}(W)=0$.

Then

$$
|\operatorname{supp} V| \leq \begin{cases}2 m-1 & \text { if } \operatorname{supp} V \text { and } \operatorname{supp} W \text { commute } \\ 2 m & \text { otherwise. }\end{cases}
$$

Proof. By (2), there exist $r_{1}, \ldots, r_{m}, p_{1} \ldots, p_{m} \in \operatorname{supp} V, s^{\prime}, p_{m+1} \in \operatorname{supp} W$ and $t \in Q_{m}\left(r_{1}, \ldots, r_{m}, s^{\prime}\right)$ such that

$$
\left(p_{1}, \ldots, p_{m+1}\right) \in \operatorname{supp} t \quad \text { and } \quad \varphi_{m+1}(v \otimes t)=0
$$

for all $p \in \operatorname{supp} V, v \in V_{p}$. Let

$$
Y=\left\{p_{j}: 1 \leq j \leq m\right\} \cup\left\{\left(p_{j+1} p_{j+2} \cdots p_{m+1}\right)^{-1} \triangleright p_{j}: 1 \leq j \leq m\right\} .
$$

Then $|Y| \leq 2 m$. Moreover, if $r \triangleright s=s$ for all $r \in \operatorname{supp} V, s \in \operatorname{supp} W$, then we have $p_{m+1}^{-1} \triangleright p_{m}=p_{m}$ and hence $|Y| \leq 2 m-1$. Therefore it suffices to prove that $Y=\operatorname{supp} V$.

By Proposition 5.5, any element $p \in \operatorname{supp} V$ with $p \notin Y$ satisfies $p_{j} \triangleright p=p$ for all $j \in\{1, \ldots, m+1\}$ and hence supp $V=Y \cup C(Y)$. By Lemma 1.11, $Y$ is Inn( $\operatorname{supp} V$ )-invariant. Thus $Y=\operatorname{supp} V$ if $\operatorname{supp} V$ is indecomposable. If $\operatorname{supp} V$ is decomposable as in (1), then $p_{m}$ and $p_{m+1}^{-1} \triangleright p_{m}$ are in different components of $\operatorname{supp} V$ by (1). Therefore the $\operatorname{Inn}(\operatorname{supp} V)$-invariance of $Y$ implies again that $Y=\operatorname{supp} V$.

Corollary 5.8. Let $r_{1}, r_{2}, r_{3}, r_{4} \in \operatorname{supp} V$ and $s \in \operatorname{supp} W$. Assume that supp $V$ and supp $W$ commute, $\left(r_{3}, r_{4}, s\right) \in \operatorname{supp} Q_{2}\left(r_{3}, r_{4}, s\right)$, and

$$
\begin{array}{ll}
r_{2} \notin\left\{r_{3}, r_{4}, r_{4}^{-1} \triangleright r_{3}\right\}, & r_{2} \triangleright r_{4} \neq r_{4}, \\
r_{1} \notin\left\{r_{2} \triangleright r_{3}, r_{2}, r_{4}, r_{4}^{-1} \triangleright r_{2}, r_{4}^{-1} \triangleright r_{3}\right\}, & r_{1} \triangleright r_{4} \neq r_{4} .
\end{array}
$$

Then $(\operatorname{ad} V)^{4}(W) \neq 0$. 
Proof. Let $\left(p_{1}, p_{2}, p_{3}\right)=\left(r_{3}, r_{4}, s\right) \in \operatorname{supp} Q_{2}\left(r_{3}, r_{4}, s\right)$. By assumption, conditions (5.1), (5.2) with $m=i=2, p=r_{2}$ are fulfilled:

- $r_{4} \triangleright r_{2} \neq r_{2}$,

- $s \triangleright r_{2}=r_{2}$,

- $r_{2} \notin\left\{r_{3}, r_{4}, r_{4}^{-1} \triangleright r_{3}\right\}$.

Hence $\left(r_{2} \triangleright r_{3}, r_{2}, r_{4}, s\right) \in \operatorname{supp} Q_{3}\left(r_{2}, r_{3}, r_{4}, s\right)$ by Proposition 5.5.

Now let $\left(p_{1}, p_{2}, p_{3}, p_{4}\right)=\left(r_{2} \triangleright r_{3}, r_{2}, r_{4}, s\right) \in \operatorname{supp} Q_{3}\left(r_{2}, r_{3}, r_{4}, s\right)$. Then

$$
\left(p_{3} p_{4}\right)^{-1} \triangleright p_{2}=r_{4}^{-1} \triangleright r_{2}, \quad\left(p_{2} p_{3} p_{4}\right)^{-1} \triangleright p_{1}=r_{4}^{-1} \triangleright r_{3} .
$$

By assumption, conditions (5.1), (5.2) with $m=i=3, p=r_{1}$ are fulfilled:

- $p_{3} \triangleright p \neq p$,

- $p_{4} \triangleright p=p$,

- $p \notin\left\{p_{1}, p_{2}, p_{3},\left(p_{3} p_{4}\right)^{-1} \triangleright p_{2},\left(p_{2} p_{3} p_{4}\right)^{-1} \triangleright p_{1}\right\}$.

Hence $\left(r_{1} r_{2} \triangleright r_{3}, r_{1} \triangleright r_{2}, r_{1}, r_{4}, s\right) \in \operatorname{supp} Q_{4}\left(r_{1}, r_{2}, r_{3}, r_{4}, s\right)$ and therefore we have $(\operatorname{ad} V)^{4}(W) \neq 0$ by Remark 5.4.

Corollary 5.9. Assume that

- $|\operatorname{supp} V| \geq 5$,

- $\operatorname{supp} V$ and supp $W$ commute,

- $(\operatorname{ad} V)(W) \neq 0$,

- $x \triangleright y \neq y$ for all $x, y \in \operatorname{supp} V$.

Then $(\operatorname{ad} V)^{4}(W) \neq 0$.

Proof. Since $(\operatorname{ad} V)(W) \neq 0$, there exist elements $r_{4} \in \operatorname{supp} V$ and $s \in \operatorname{supp} W$ such that $Q_{1}\left(r_{4}, s\right) \neq 0$. Then $\left(r_{4}, s\right)=\operatorname{supp} Q_{1}\left(r_{4}, s\right)$. Let $r_{3} \in \operatorname{supp} V \backslash\left\{r_{4}\right\}$. Then $r_{3} \triangleright r_{4} \neq r_{4}$ and hence $\left(r_{3}, r_{4}, s\right) \in \operatorname{supp} Q_{2}\left(r_{3}, r_{4}, s\right)$ by Proposition 5.5 . Since $|\operatorname{supp} V| \geq 5$, there exists an $r_{2} \in \operatorname{supp} V$ with $r_{2} \notin\left\{r_{3}, r_{4}, r_{4}^{-1} \triangleright r_{3}, r_{4} \triangleright r_{3}\right\}$. By assumption, $r_{2} \triangleright r_{4} \neq r_{4}$. By construction,

$$
r_{3} \notin\left\{r_{2} \triangleright r_{3}, r_{2}, r_{4}, r_{4}^{-1} \triangleright r_{2}, r_{4}^{-1} \triangleright r_{3}\right\}, \quad r_{3} \triangleright r_{4} \neq r_{4} .
$$

Thus Corollary 5.8 with $r_{1}=r_{3}$ implies that $(\operatorname{ad} V)^{4}(W) \neq 0$.

Corollary 5.10. Let $r_{1}, r_{2}, r_{3} \in \operatorname{supp} V$ and $s \in \operatorname{supp} W$. Assume that the following hold:

(1) $r_{2} \triangleright r_{3} \neq r_{3}$,

(2) $r_{1} \notin\left\{r_{3} r_{2} \triangleright r_{3}, r_{3} \triangleright r_{2}, r_{3}, s^{-1} \triangleright r_{3}, s^{-1} \triangleright r_{2}\right\}$, 
(3) $s \triangleright r_{2}, s \triangleright r_{3} \notin\left\{r_{2}, r_{3}\right\}$,

(4) $r_{1} \triangleright s \neq s$ or $r_{1} \triangleright r_{3} \neq r_{3}$.

Then $(\operatorname{ad} V)^{4}(W) \neq 0$.

Proof. Let $\left(p_{1}, p_{2}\right)=\left(r_{3}, s\right)$. Then $\left(p_{1}, p_{2}\right) \in \operatorname{supp} Q_{1}\left(r_{3}, s\right)$ since $s \triangleright r_{3} \neq r_{3}$. Conditions (5.1), (5.2) with $m=1, i=2$ and $p=r_{2}$ are fulfilled: $p_{2} \triangleright p \neq p$ and $p=r_{2} \notin\left\{r_{3}, s^{-1} \triangleright r_{3}\right\}$. Thus we have $\left(r_{2} \triangleright r_{3}, r_{2}, s\right) \in \operatorname{supp} Q_{2}\left(r_{2}, r_{3}, s\right)$ by Proposition 5.5.

Let $\left(p_{1}, p_{2}, p_{3}\right)=\left(r_{2} \triangleright r_{3}, r_{2}, s\right) \in \operatorname{supp} Q_{2}\left(r_{2}, r_{3}, s\right)$. Then conditions (5.1) and (5.2) with $m=2, i=3$ and $p=r_{3}$ are fulfilled: $s \triangleright p \neq p$,

$r_{3} \notin\left\{p_{1}, p_{2}, p_{3}^{-1} \triangleright p_{2},\left(p_{2} p_{3}\right)^{-1} \triangleright p_{1}\right\}=\left\{r_{2} \triangleright r_{3}, r_{2}, s^{-1} \triangleright r_{2}, s^{-1} \triangleright r_{3}\right\}$.

Hence $\left(r_{3} r_{2} \triangleright r_{3}, r_{3} \triangleright r_{2}, r_{3}, s\right) \in \operatorname{supp} Q_{3}\left(r_{3}, r_{2}, r_{3}, s\right)$ by Proposition 5.5.

Finally, let $\left(p_{1}, p_{2}, p_{3}, p_{4}\right)=\left(r_{3} r_{2} \triangleright r_{3}, r_{3} \triangleright r_{2}, r_{3}, s\right) \in \operatorname{supp} Q_{3}\left(r_{3}, r_{2}, r_{3}, s\right)$ and let $p=r_{1}$. Then

$$
\begin{aligned}
p_{4}^{-1} \triangleright p_{3} & =s^{-1} \triangleright r_{3}, \\
\left(p_{3} p_{4}\right)^{-1} \triangleright p_{2} & =s^{-1} \triangleright r_{2}, \\
\left(p_{2} p_{3} p_{4}\right)^{-1} \triangleright p_{1} & =s^{-1} \triangleright r_{3}
\end{aligned}
$$

and hence

$$
p \notin\left\{p_{1}, p_{2}, p_{3}, p_{4}^{-1} \triangleright p_{3},\left(p_{3} p_{4}\right)^{-1} \triangleright p_{2},\left(p_{2} p_{3} p_{4}\right)^{-1} \triangleright p_{1}\right\} .
$$

Since $p_{4} \triangleright p \neq p$ or $p_{3} \triangleright p \neq p$ by (4), Proposition 5.5 with $m=3$ implies that $Q_{4}\left(r_{1}, r_{3}, r_{2}, r_{3}, s\right) \neq 0$. Hence $(\operatorname{ad} V)^{4}(W) \neq 0$ by Lemma 5.2 .

Corollary 5.11. Assume that

- $\operatorname{supp} V$ is an indecomposable quandle,

- $(\operatorname{ad} V)(W) \neq 0,(\operatorname{ad} V)^{4}(W)=0$.

Then $\operatorname{supp} V$ is isomorphic to one of the following quandles:

$$
\{1\},(12)^{\mathbb{S}_{3}},(12)^{\mathbb{S}_{4}},(123)^{\mathbb{A}_{4}}, \operatorname{Aff}(5,2), \operatorname{Aff}(5,3), \operatorname{Aff}(5,4),(1234)^{\mathbb{S}_{4}} .
$$

Proof. Corollary 5.7 yields that $|\operatorname{supp} V| \leq 6$ and Remark 1.6 applies.

\subsection{Commuting supports}

Let $g, h \in G$. Assume that $\operatorname{supp} V=g^{G}$, supp $W=h^{G}, G=\left\langle g^{G} \cup h^{G}\right\rangle$ and that $g^{G}$ and $h^{G}$ commute. We conclude an implication of $(\operatorname{ad} V)^{2}(W)=0$ and $(\operatorname{ad} W)^{4}(V)=0$ on $V$ and $W$. 
Lemma 5.12. The quandles $g^{G}$ and $h^{G}$ are indecomposable.

Proof. It is sufficient to prove the claim on $h^{G}$. By Lemma 5.1 and since $g^{G}$ and $h^{G}$ commute, we obtain that

$$
h^{G}=G \triangleright h=\left\langle h^{G}\right\rangle\left\langle g^{G}\right\rangle \triangleright h=\left\langle h^{G}\right\rangle \triangleright h=\operatorname{Inn}\left(h^{G}\right) \triangleright h .
$$

Thus $h^{G}$ is indecomposable.

Lemma 5.13. Assume that $(\operatorname{ad} V)(W) \neq 0$ and $(\operatorname{ad} V)^{2}(W)=0$. Then $g^{G}=\{g\}$.

Proof. This follows from Corollary 5.7 with $m=1$ using Lemma 5.12.

Proposition 5.14. Assume that

$$
(\operatorname{ad} V)(W) \neq 0, \quad(\operatorname{ad} V)^{2}(W)=0 \quad \text { and } \quad(\operatorname{ad} W)^{4}(V)=0 .
$$

Then $g^{G} \cup h^{G}$ is isomorphic to $Z_{3}^{3,1}$ or $Z_{T}^{4,1}$.

Proof. First, we have $g^{G}=\{g\}$ by Lemma 5.13. Further, $h^{G}$ is indecomposable by Lemma 5.12. and $\left|h^{G}\right| \geq 2$ since $G=\left\langle g^{G} \cup h^{G}\right\rangle$ is non-abelian. Corollary 5.7 implies that $\left|h^{G}\right| \leq 5$. Thus, by Corollary $5.11, h^{G}$ is isomorphic to one of the quandles $(12)^{\mathbb{S}_{3}},(123)^{\mathbb{A}_{4}}, \operatorname{Aff}(5,2), \operatorname{Aff}(5,3), \operatorname{Aff}(5,4)$. Assume that $h^{G}$ is one of the quandles $\operatorname{Aff}(5,2), \operatorname{Aff}(5,3), \operatorname{Aff}(5,4)$. Then $\left|h^{G}\right|=5$ and $x \triangleright y \neq y$ for any $x, y \in h^{G}$ with $x \neq y$. Thus $(\operatorname{ad} W)^{4}(V) \neq 0$ by Corollary 5.9 and the proposition follows.

\subsection{Non-commuting supports}

In this subsection let $g, h \in G$. Assume that $g h \neq h g$, $\operatorname{supp} V=g^{G}$, supp $W=h^{G}$ and $G=\left\langle g^{G} \cup h^{G}\right\rangle$. Then for all $s \in h^{G}$ there exists an element $r \in g^{G}$ such that $r s \neq s r$. We determine some consequences of the equations $(\operatorname{ad} V)^{2}(W)=0$ and $(\operatorname{ad} W)^{4}(V)=0$.

Lemma 5.15. Assume that $(\operatorname{ad} V)^{2}(W)=0$. Then the following hold:

(1) $g^{G}$ is commutative.

(2) $g^{G} \neq h^{G}$.

(3) $g^{G}=\left\langle h^{G}\right\rangle \triangleright g$.

(4) Let $s \in h^{G}$. Then there exist $r_{1}, r_{2} \in g^{G}$ such that $\left.\varphi_{s}\right|_{g} G=\left(r_{1} r_{2}\right)$.

(5) $h^{2} \triangleright g=g$ and $(g h)^{2}=(h g)^{2}$.

(6) For all $m \in \mathbb{Z},\left\{x \in g^{G}: x \triangleright\left(g^{m} \triangleright h\right) \neq g^{m} \triangleright h\right\}=\{g, h \triangleright g\}$. 
Proof. (4) and (1) First, $\left|g^{G}\right| \geq 2$ and $\left|h^{G}\right| \geq 2$ since $g h \neq h g$. Let $r_{1} \in g^{G}$ and $s \in h^{G}$ such that $s \triangleright r_{1} \neq r_{1}$. Then $\left(r_{1}, s\right) \in \operatorname{supp} Q_{1}\left(r_{1}, s\right)$ by Remark 5.6. Let $p \in g^{G}$. Assume that $p \notin\left\{r_{1}, s^{-1} \triangleright r_{1}\right\}$. Since $Q_{2}\left(p, r_{1}, s\right)=0$ because of $(\operatorname{ad} V)^{2}(W)=0$, Proposition 5.5 implies that $s \triangleright p=p=r_{1} \triangleright p$. Then

$$
\left.\varphi_{s}\right|_{g G}=\left(r_{1} s^{-1} \triangleright r_{1}\right)
$$

which is the claim in (4). The equation $r_{1} \triangleright p=p$ implies that $r_{1} \triangleright r_{2}=r_{2}$ for all $r_{2} \in g^{G}$. Thus (1) holds.

(2) If $g^{G}=h^{G}$, then $G=\left\langle g^{G}\right\rangle$ is commutative by (1), a contradiction.

(3) Lemma 5.1 and (1) yield that

$$
g^{G}=G \triangleright g=\left\langle h^{G}\right\rangle\left\langle g^{G}\right\rangle \triangleright g=\left\langle h^{G}\right\rangle \triangleright g .
$$

(5) From (1) we know that $g \triangleright(h \triangleright g)=h \triangleright g$ and hence

$$
h g h \triangleright g=h^{2} \triangleright g=g,
$$

where the second equation follows from (4). This implies (5).

(6) By (1), $g^{G}$ is commutative. Thus it suffices to prove the claim for $m=0$. The latter follows from (4) with $s=h$ since $g h \neq h g$.

Lemma 5.16. Assume that $(\operatorname{ad} V)^{2}(W)=0$ and that $h$ commutes with $g \triangleright h$. Then the following hold:

(1) For all $m \in \mathbb{Z},(h \triangleright g) \triangleright\left(g^{m} \triangleright h\right)=g^{m+1} \triangleright h$.

(2) $\left\langle g^{G}\right\rangle \triangleright h=\langle g\rangle \triangleright h$.

Proof. First we prove (1). By Lemma 5.15 (1), $g^{G}$ is commutative. Thus it suffices to consider the case $m=0$. Now $(h \triangleright g) \triangleright h=h g \triangleright h=g \triangleright h$ by assumption.

Now we prove (2). Lemma 5.15 (4) and (1) with $m \in\{-1,0\}$, imply that

$$
\left(g^{G}\right)^{ \pm 1} \triangleright h \subseteq\{h\} \cup\left\{g, g^{-1}, h \triangleright g,(h \triangleright g)^{-1}\right\} \triangleright h \subseteq\left\{h, g \triangleright h, g^{-1} \triangleright h\right\} .
$$

Now write $\left\langle g^{G}\right\rangle=\bigcup_{m \in \mathbb{N}_{0}} A_{m}$, where $A_{m}=\left\{x_{1}^{ \pm 1} \cdots x_{m}^{ \pm 1}: x_{i} \in g^{G}\right\}$. It suffices to show that $A_{m} \triangleright h \subseteq\langle g\rangle \triangleright h$ for all $m \in \mathbb{N}_{0}$. We proceed by induction on $m$. The case $m=0$ is trivial and the case $m=1$ was just proven. Let now $m \in \mathbb{N}$ and assume that $A_{m} \triangleright h \subseteq\langle g\rangle \triangleright h$. Using the induction hypothesis and the fact that $g^{G}$ is commutative, see Lemma 5.15 (1), we obtain that

$$
\begin{aligned}
A_{m+1} \triangleright h & =\left(g^{G}\right)^{ \pm 1} \triangleright\left(A_{m} \triangleright h\right) \\
& \subseteq\left(g^{G}\right)^{ \pm 1} \triangleright(\langle g\rangle \triangleright h)=\langle g\rangle \triangleright\left(\left(g^{G}\right)^{ \pm 1} \triangleright h\right) \subseteq\langle g\rangle \triangleright h .
\end{aligned}
$$

This implies (2). 
Lemma 5.17. Assume that $(\operatorname{ad} V)^{2}(W)=0$ and $h^{G}$ is commutative. Then $g^{G} \cup h^{G}$ is isomorphic to $Z_{2}^{2,2}$.

Proof. Lemma 5.15(4) implies that $g h g \triangleright h=h$. Since $h^{G}$ is commutative, we have $h g \triangleright h=g \triangleright h$ and hence $h=g h g \triangleright h=g^{2} \triangleright h$. Therefore

$$
h^{G}=\left\langle g^{G}\right\rangle\left\langle h^{G}\right\rangle \triangleright h=\left\langle g^{G}\right\rangle \triangleright h=\{h, g \triangleright h\}
$$

by Lemmas 5.1 and $5.16(2)$ and since $h^{G}$ is commutative. Recall that $g^{G}$ is commutative by Lemma 5.15(1) and that $h^{2} \triangleright g=g$ by Lemma 5.15(5). From Lemma 5.1 we obtain

$$
g^{G}=\left\langle h^{G}\right\rangle\left\langle g^{G}\right\rangle \triangleright g=\left\langle h^{G}\right\rangle \triangleright g=\langle h, g \triangleright h\rangle \triangleright g \subseteq\langle g, h\rangle \triangleright g=\{h \triangleright g, g\} .
$$

Therefore $g^{G}=\{h \triangleright g, g\}$ and $g^{G} \cup h^{G} \simeq Z_{2}^{2,2}$ as quandles.

Lemma 5.18. Let $x, y \in h^{G}$ such that $x \triangleright y=y$. Assume that

- $y \triangleright z \neq z$ for all $z \in h^{G} \backslash\{x, y\}$,

- $\left.\varphi_{x}\right|_{g^{G}}=(r s)$ for some $r, s \in g^{G}, r \neq s$.

Then $\left.\varphi_{y}\right|_{g} G=(r s)$.

Proof. Since $x, y \in h^{G}$ and $\left.\varphi_{x}\right|_{g^{G}}=(r s)$, there exist elements $a, b \in g^{G}$ such that $\left.\varphi_{y}\right|_{g^{G}}=(a b)$. Assume that $(a b) \neq(r s)$. Then $|\{r, s, a, b\}|=4$ since $\left.\varphi_{x}\right|_{g G}$ and $\left.\varphi_{y}\right|_{g G}$ commute. Let $z=r \triangleright x$. First, $z=r \triangleright x \neq x$ since $x \triangleright r \neq r$. Second, $r \triangleright x \neq y$ since $\left.\varphi_{z}\right|_{g G}=(r r \triangleright s) \neq(a b)$. Hence $y \triangleright z \neq z$ by assumption, a contradiction to $y \triangleright(r \triangleright x)=(y \triangleright r) \triangleright(y \triangleright x)=r \triangleright x$.

Lemma 5.19. Let $x, y \in h^{G}$ be elements such that $\psi_{x}=\left.\varphi_{x}\right|_{g G}$ and $\psi_{y}=\left.\varphi_{y}\right|_{g}$. Assume that $(\operatorname{ad} V)^{2}(W)=0$ and that $x, y$ generate the quandle $h^{G}$. If $\psi_{x}=\psi_{y}$, then $\left|g^{G}\right|=2$. Otherwise $\left|g^{G}\right|=3$ and $\psi_{x} \psi_{y} \neq \psi_{y} \psi_{x}$.

Proof. By Lemma 5.15 (1) and (4), $g^{G}$ is commutative and there exist $g_{1}, g_{2} \in g^{G}$ such that $\psi_{x}=\left(g_{1} x \triangleright g_{1}\right)$ and $\psi_{y}=\left(g_{2} y \triangleright g_{2}\right)$. Assume now that

$$
\left|\left\{g_{1}, x \triangleright g_{1}, g_{2}, y \triangleright g_{2}\right\}\right|=4 \text {. }
$$

Then $\left|g^{G}\right| \geq 4$. On the other hand, Lemma 5.1 and the commutativity of $g^{G}$ imply that

$$
g^{G}=G \triangleright g_{1}=\left\langle h^{G}\right\rangle\left\langle g^{G}\right\rangle \triangleright g_{1}=\left\langle h^{G}\right\rangle \triangleright g_{1}=\langle x, y\rangle \triangleright g_{1}=\left\{g_{1}, x \triangleright g_{1}\right\},
$$

a contradiction to $\left|g^{G}\right| \geq 4$. Hence $\left|\left\{g_{1}, x \triangleright g_{1}, g_{2}, y \triangleright g_{2}\right\}\right| \leq 3$ and the lemma follows by two calculations similar to (5.9). 
Lemma 5.20. Assume that $(\operatorname{ad} V)^{2}(W)=0$ and that $h^{G}$ is decomposable. Let $h^{G}=Y_{1} \cup \cdots \cup Y_{k}$ be the decomposition of $h^{G}$ into orbits of the inner group of $h^{G}$. Then $k=2$ and $x \triangleright Y_{1}=Y_{2}, x \triangleright Y_{2}=Y_{1}$ for all $x \in g^{G}$.

Proof. First, $h^{G}=g \triangleright h^{G}=\left(g \triangleright Y_{1}\right) \cup \cdots \cup\left(g \triangleright Y_{k}\right)$ is a decomposition into $\operatorname{Inn}\left(h^{G}\right)$-orbits:

$$
(g \triangleright y) \triangleright\left(g \triangleright Y_{i}\right)=g \triangleright\left(y \triangleright Y_{i}\right)=g \triangleright Y_{i} \quad \text { for all } y \in h^{G}, 1 \leq i \leq k .
$$

Thus $\varphi_{g}$ permutes the orbits $Y_{1}, \ldots, Y_{k}$. Since $g^{G}=\left\langle h^{G}\right\rangle \triangleright g$ by Lemma 5.15 (3), each $x \in g^{G}$ permutes the $\operatorname{Inn}\left(h^{G}\right)$-orbits $Y_{1}, \ldots, Y_{k}$ in the same way as $g$ does. Let $Y \subset h^{G}$ be the $\operatorname{Inn}\left(h^{G}\right)$-orbit of $h$. As $G$ is generated by $g^{G} \cup h^{G}$ and $h^{G}$ is a conjugacy class of $G$, we conclude that

$$
h^{G}=Y \cup(g \triangleright Y) \cup \cdots \cup\left(g^{k-1} \triangleright Y\right) .
$$

By Lemma 5.15 (5), $g h g \triangleright h=h$ and hence $h \in Y \cap\left(g^{2} \triangleright Y\right)$. Thus $g^{2} \triangleright Y=Y$ and hence $h^{G}=Y \cup(g \triangleright Y)$ since $h^{G}$ is decomposable.

Lemma 5.21. Assume that $(\operatorname{ad} V)^{2}(W)=0$ and $(\operatorname{ad} W)^{4}(V)=0$ and that $h^{G}$ is decomposable. Then $g^{G} \cup h^{G}$ is isomorphic to $Z_{2}^{2,2}$ or to $Z_{4}^{4,2}$.

Proof. By Lemma 5.20, $h^{G}=Y_{1} \cup Y_{2}$, where $Y_{1}$ and $Y_{2}$ are the $\operatorname{Inn}\left(h^{G}\right)$-orbits of $h^{G}$. Moreover, $x \triangleright Y_{1}=Y_{2}$ and $x \triangleright Y_{2}=Y_{1}$ for all $x \in g^{G}$. Thus Corollary 5.7 implies that $\left|h^{G}\right| \leq 6$. There are two cases to consider.

Assume first that $Y_{1}$ is non-commutative. Then we have $Y_{1} \simeq Y_{2} \simeq(12)^{\mathbb{S}_{3}}$ by Remark 1.5. Let $r_{3} \in Y_{1}, r_{2} \in Y_{1} \backslash\left\{r_{3}\right\}$ and $r_{1} \in Y_{2} \backslash\left\{g^{-1} \triangleright r_{3}, g^{-1} \triangleright r_{2}\right\}$. By Corollary 5.10 with $s=g$, $(\operatorname{ad} W)^{4}(V) \neq 0$, a contradiction.

Assume now that $Y_{1}$ is commutative. By Lemma 1.12, the permutations $\varphi_{i}$ defining $h^{G}$ are given by (1.2). Further, $x \triangleright y \neq y$ and hence $y \triangleright x \neq x$ for all $x \in g^{G}, y \in h^{G}$. But $\left.\varphi_{y}\right|_{g^{G}}$ is a transposition for all $y \in h^{G}$ by Lemma 5.15 (4), and hence $\left|g^{G}\right|=2$.

If $\left|Y_{1}\right|=1$, then $h^{G}$ is commutative and $g^{G} \cup h^{G} \simeq Z_{2}^{2,2}$ by Lemma 5.17. Suppose next that $\left|Y_{1}\right|=2$. Then we have $h^{G} \simeq Z_{2}^{2,2}$ by Lemma 1.12. Let $h^{\prime} \in Y_{1}$ with $h^{\prime} \neq h$. Since $g h g \triangleright h=h$ by Lemma 5.15(5) and since $h g \triangleright h \neq g \triangleright h$, we conclude that $g^{2} \triangleright h \neq h, \varphi_{g}=\left(h g \triangleright h h^{\prime} g \triangleright h^{\prime}\right)$, and $\varphi_{h \triangleright g}=\varphi_{g}^{-1}$. Therefore $g^{G} \cup h^{G} \simeq Z_{4}^{4,2}$.

Finally, assume that $\left|Y_{1}\right|=3$. Let $r_{2} \in Y_{1}$. Then $r_{2} \triangleright x \neq x$ for all $x \in Y_{2}$, by Lemma 1.12. Take $r_{3} \in Y_{2} \backslash\left\{g \triangleright r_{2}, g^{-1} \triangleright r_{2}\right\}$ and $r_{1} \in Y_{1} \backslash\left\{r_{3} \triangleright r_{2}, g^{-1} \triangleright r_{3}\right\}$. Then $(\operatorname{ad} W)^{4}(V) \neq 0$ by Corollary 5.10 with $s=g$, a contradiction.

Lemma 5.22. Assume that $h^{2} \triangleright g=g$. Then $h^{2} \triangleright(g \triangleright h)=g \triangleright h$. In particular, $h^{G}$ is not isomorphic to any of $(123)^{\mathbb{A} 4}, \operatorname{Aff}(5,2)$ and $\operatorname{Aff}(5,3)$. 
Proof. The first claim follows from the definition of a quandle. Since $h$ and $g \triangleright h$ are fixed points of $\left.\varphi_{h}^{2}\right|_{h^{G}}$, the second claim follows from Remark 1.6.

Lemma 5.23. Assume that $(\operatorname{ad} V)^{2}(W)=0$ and $(\operatorname{ad} W)^{4}(V)=0$. Then $h^{G}$ is not isomorphic to $\operatorname{Aff}(5,4)$.

Proof. Assume that $h^{G} \simeq \operatorname{Aff}(5,4)$. Then $h^{G}$ can be generated by two elements $x$ and $y$ with $x \neq y$. By Lemma 5.15(4), $\left.\varphi_{x}\right|_{g^{G}}$ and $\left.\varphi_{y}\right|_{g^{G}}$ are transpositions. By Lemma 5.19, either $\left|g^{G}\right|=2$ or $\left|g^{G}\right|=3,\left.\varphi_{x}\right|_{g^{G}} \neq\left.\varphi_{y}\right|_{g^{G}}$. Assume the second case. Let $z \in g^{G}$ such that $x \triangleright z \neq z, y \triangleright z \neq z$. Then

$$
x \triangleright z \neq y \triangleright z, \quad x \triangleright(y \triangleright z)=y \triangleright z, \quad y \triangleright(x \triangleright z)=x \triangleright z .
$$

Therefore

$$
x y x y x \triangleright z=y \triangleright z \neq x \triangleright z=y x y x y \triangleright z,
$$

a contradiction to $x y x y x=y x y x y$ in $G$. Hence $\left|g^{G}\right|=2$.

Now $g \triangleright z \neq z$ for all $z \in h^{G}$ and therefore we may assume that $g^{3} \triangleright h \neq h$. Moreover, for all $z_{1}, z_{2} \in h^{G}$ there exists an element $z \in h^{G}$ such that $z \triangleright z_{1}=z_{2}$. So let $r_{2} \in h^{G}$ such that $r_{2} \triangleright h=g \triangleright h$ and let $r_{3}=h$. Since $g h g h=h g h g$ by Lemma 5.15(5), we conclude that $r_{2} \triangleright r_{3} \neq r_{3}, g \triangleright r_{2} \neq r_{2}, g \triangleright r_{2} \neq r_{3}$ since

$$
\left(g \triangleright r_{2}\right) \triangleright(g \triangleright h)=g \triangleright\left(r_{2} \triangleright h\right)=g^{2} \triangleright h \neq g^{-1} \triangleright h=h \triangleright(g \triangleright h)
$$

and $g \triangleright r_{3} \notin\left\{r_{2}, r_{3}\right\}$. Moreover, $r_{3} r_{2} \triangleright r_{3}=h g \triangleright h=g^{-1} \triangleright r_{3}$, and hence there exists an $r_{1} \in h^{G} \backslash\left\{r_{3} r_{2} \triangleright r_{3}, r_{3} \triangleright r_{2}, r_{3}, g^{-1} \triangleright r_{2}, g^{-1} \triangleright r_{3}\right\}$. Since $r_{1} \triangleright g \neq g$, Corollary 5.10 with $s=g$ implies that $(\operatorname{ad} W)^{4}(V) \neq 0$. This is a contradiction and hence $h^{G} \not \operatorname{Aff}(5,4)$.

Lemma 5.24. Assume that $(\operatorname{ad} V)^{2}(W)=0$ and $(\operatorname{ad} W)^{4}(V)=0$. Then $h^{G}$ is neither isomorphic to $(1234)^{\mathbb{S}_{4}}$ nor to $(12)^{\mathbb{S}_{4}}$.

Proof. Assume that $h^{G} \simeq(1234)^{\mathbb{S}_{4}}$ or $h^{G} \simeq(12)^{\mathbb{S}_{4}}$. Let $r_{3} \in h^{G}$ and $s \in g^{G}$ with $s \triangleright r_{3} \neq r_{3}$, and let $x \in h^{G} \backslash\left\{r_{3}\right\}$ with $r_{3} \triangleright x=x$. It suffices to show that $s \triangleright r_{3}=x, s \triangleright x=r_{3}$, and $\left.\varphi_{s}\right|_{h^{G}} \neq\left(x r_{3}\right)$. Indeed, let $r_{2} \in h^{G} \backslash\left\{r_{3}, x\right\}$ with $s \triangleright r_{2} \neq r_{2}$ and let $r_{1} \in h^{G} \backslash\left\{r_{3} r_{2} \triangleright r_{3}, r_{3} \triangleright r_{2}, r_{3}, s^{-1} \triangleright r_{3}, s^{-1} \triangleright r_{2}\right\}$. Then we have $r_{1} \triangleright r_{3} \neq r_{3}$ since $r_{1} \neq r_{3}$ and $r_{1} \neq s^{-1} \triangleright r_{3}=x$, and hence Corollary 5.10 contradicts to $(\operatorname{ad} W)^{4}(V)=0$.

Now we show that $s \triangleright r_{3}=x$ and $s \triangleright x=r_{3}$. First, $\left.\varphi_{r_{3}}\right|_{g}$ and $\left.\varphi_{x}\right|_{g G}$ are transpositions by Lemma 5.15 (4). If $h^{G} \simeq(1234)^{\mathbb{S}_{4}}$, then $r_{3}^{2} \triangleright\left(s \triangleright r_{3}\right)=s \triangleright r_{3}$ and $\left.\varphi_{r_{3}}^{2}\right|_{h^{G}}$ has only $r_{3}$ and $x$ as fixed points. Hence we obtain $s \triangleright r_{3}=x$ and similarly $s \triangleright x=r_{3}$. If $h^{G} \simeq(12)^{\mathbb{S}_{4}}$, then Lemma 5.18 implies that $\left.\varphi_{r_{3}}\right|_{g} G=\left.\varphi_{x}\right|_{g^{G}}$. Hence $r_{3} x \triangleright\left(s \triangleright r_{3}\right)=s \triangleright r_{3}$. Since $\left.\varphi_{r_{3}} \varphi_{x}\right|_{h^{G}}$ has only $r_{3}$ and $x$ as fixed points, we conclude that $s \triangleright r_{3}=x$ and similarly $s \triangleright x=r_{3}$. 
Now we show that there exists an element $y \in h^{G} \backslash\left\{r_{3}, x\right\}$ such that $s \triangleright y \neq y$. If $h^{G} \simeq(1234)^{\mathbb{S}_{4}}$, then Lemma 5.19 implies that $\left|g^{G}\right| \leq 3$ and the claim holds. If $h^{G} \simeq(12)^{\mathbb{S}_{4}}$, then let $z \in h^{G} \backslash\left\{r_{3}, x\right\}$. Then $r_{3}, x$ and $z$ generate $h^{G}$ as a quandle. Recall that

$$
\left.\varphi_{r_{3}}\right|_{g^{G}}=\left.\varphi_{x}\right|_{g^{G}}=(s x \triangleright s) .
$$

If $\left.\varphi_{z}\right|_{g G}=(a b)$ with $|\{s, x \triangleright s, a, b\}|=4$, then $\left|g^{G}\right|=2$ by a calculation similar to (5.9) of Lemma 5.19, a contradiction. Otherwise $\left|g^{G}\right| \leq 3$ as in the proof of Lemma 5.19. Then again $y \triangleright s \neq s$ for four or six elements $y \in h^{G}$.

Proposition 5.25. Assume that $(\operatorname{ad} V)^{2}(W)=0,(\operatorname{ad} W)^{4}(V)=0$. Then $g^{G} \cup h^{G}$ is isomorphic to $Z_{2}^{2,2}, Z_{3}^{3,2}$ or $Z_{4}^{4,2}$.

Proof. First, $g^{G} \neq h^{G}$ by Lemma 5.15 (2).

If $h^{G}$ is commutative, then

$$
g^{G} \cup h^{G} \simeq Z_{2}^{2,2}
$$

by Lemma 5.17 .

If $h^{G}$ is decomposable, then

$$
g^{G} \cup h^{G} \simeq Z_{2}^{2,2} \quad \text { or } \quad g^{G} \cup h^{G} \simeq Z_{4}^{4,2}
$$

by Lemma 5.21 .

Finally, suppose that $h^{G}$ is non-commutative and indecomposable. In this case Corollary 5.11 implies that $h^{G}$ is isomorphic to one of the non-commutative quandles of (5.8). Since $h^{2} \triangleright g=g$ by Lemma 5.15 (5), Lemmas 5.22-5.24 imply that $h^{G} \simeq(12)^{\mathbb{S}_{3}}$. Then $\left|g^{G}\right|=2$ or $\left|g^{G}\right|=3$ by Lemma 5.19 and $g^{G}$ is commutative by Lemma 5.15 (1). If $\left|g^{G}\right|=2$, then $g \triangleright x \neq x$ for all $x \in h^{G}$ and hence $\varphi_{g}$ is a three-cycle and $\varphi_{h \triangleright g}=\varphi_{h} \varphi_{g} \varphi_{h}^{-1}=\varphi_{g}^{-1}$. Thus

$$
g^{G} \cup h^{G} \simeq Z_{3}^{3,2} \text {. }
$$

If $\left|g^{G}\right|=3$, then $(g \triangleright h) \triangleright g=g \triangleright(h \triangleright g)=h \triangleright g$. Then Lemma 5.15 (4) implies that

$$
\left.\varphi_{g \triangleright h}\right|_{g G}=\left.\varphi_{h}\right|_{g G}=(g h \triangleright g),
$$

a contradiction to Lemma 5.19 and $\left|g^{G}\right|=3$.

\subsection{The proof of Theorem 4.4}

Let $g \in \operatorname{supp} V, h \in \operatorname{supp} W$. Then $\operatorname{supp} V=g^{G}$, supp $W=h^{G}$ by assumption. Let $X=g^{G} \cup h^{G}$. If $g^{G}$ and $h^{G}$ commute, then

$$
X \simeq Z_{3}^{3,1} \text { or } \quad X \simeq Z_{T}^{4,1}
$$


by Proposition 5.14. Otherwise, $g^{G}$ and $h^{G}$ do not commute and

$$
X \simeq Z_{2}^{2,2} \text { or } \quad X \simeq Z_{4}^{4,2} \text { or } \quad X \simeq Z_{3}^{3,2}
$$

by Proposition 5.25. The enveloping groups of the quandles $Z_{T}^{4,1}, Z_{2}^{2,2}, Z_{3}^{3,1}$, $Z_{3}^{3,2}$ and $Z_{4}^{4,2}$ were computed in Section 2. Hence the theorem follows from the universal property of the enveloping group, see Remark 1.7.

\section{Bibliography}

[1] N. Andruskiewitsch, F. Fantino, M. Graña and L. Vendramin, Finite-dimensional pointed Hopf algebras with alternating groups are trivial, Ann. Mat. Pura Appl. (4) 190 (2011), no. 2, 225-245.

[2] N. Andruskiewitsch, F. Fantino, M. Graña and L. Vendramin, Pointed Hopf algebras over the sporadic simple groups, J. Algebra 325 (2011), 305-320.

[3] N. Andruskiewitsch and M. Graña, From racks to pointed Hopf algebras, Adv. Math. 178 (2003), no. 2, 177-243.

[4] N. Andruskiewitsch, I. Heckenberger and H.-J. Schneider, The Nichols algebra of a semisimple Yetter-Drinfeld module, Amer. J. Math. 132 (2010), no. 6, 1493-1547.

[5] N. Andruskiewitsch and H.-J. Schneider, Lifting of quantum linear spaces and pointed Hopf algebras of order $p^{3}$, J. Algebra 209 (1998), no. 2, 658-691.

[6] N. Andruskiewitsch and H.-J. Schneider, Finite quantum groups and Cartan matrices, Adv. Math. 154 (2000), no. 1, 1-45.

[7] N. Andruskiewitsch and H.-J. Schneider, On the classification of finite-dimensional pointed Hopf algebras, Ann. of Math. (2) 171 (2010), no. 1, 375-417.

[8] I. Angiono, A presentation by generators and relations of Nichols algebras of diagonal type and convex orders on root systems, J. Eur. Math. Soc. (JEMS), to appear.

[9] I. Angiono, On Nichols algebras of diagonal type, J. Reine Angew. Math. 683 (2013), 189-251.

[10] M. Cuntz and I. Heckenberger, Weyl groupoids of rank two and continued fractions, Algebra Number Theory 3 (2009), no. 3, 317-340.

[11] M. Graña, I. Heckenberger and L. Vendramin, Nichols algebras of group type with many quadratic relations, Adv. Math. 227 (2011), no. 5, 1956-1989.

[12] P. Hall, The classification of prime-power groups, J. Reine Angew. Math. 182 (1940), 130-141.

[13] I. Heckenberger, The Weyl groupoid of a Nichols algebra of diagonal type, Invent. Math. 164 (2006), no. 1, 175-188.

[14] I. Heckenberger, Classification of arithmetic root systems, Adv. Math. 220 (2009), no. $1,59-124$. 
[15] I. Heckenberger and H.-J. Schneider, Nichols algebras over groups with finite root system of rank two I, J. Algebra 324 (2010), no. 11, 3090-3114.

[16] I. Heckenberger and H.-J. Schneider, Root systems and Weyl groupoids for Nichols algebras, Proc. Lond. Math. Soc. (3) 101 (2010), no. 3, 623-654.

[17] I. Heckenberger and L. Vendramin, Nichols algebras over groups with finite root system of rank two III, preprint (2013), http://arxiv .org/abs/1309.4634.

[18] I. Heckenberger and L. Vendramin, Nichols algebras over groups with finite root system of rank two IV, preprint (2013), http: //arxiv .org/abs/1311.2881.

[19] I. Heckenberger and H. Yamane, A generalization of Coxeter groups, root systems, and Matsumoto's theorem, Math. Z. 259 (2008), no. 2, 255-276.

[20] V. K. Kharchenko, A quantum analog of the Poincaré-Birkhoff-Witt theorem (in Russian), Algebra Logika 38 (1999), no. 4, 476-507; translation in Algebra Logic 38 (1999), no. 4, 259-276.

[21] G. Lusztig, Introduction to Quantum Groups, Modern Birkhäuser Class., Birkhäuser, Boston, 2010.

[22] D. M. Rocke, p-groups with abelian centralizers, Proc. Lond. Math. Soc. (3) 30 (1975), 55-75.

[23] M. Rosso, Quantum groups and quantum shuffles, Invent. Math. 133 (1998), no. 2, 399-416.

[24] L. Vendramin, On the classification of quandles of low order, J. Knot Theory Ramifications 21 (2012), no. 9, 1250088.

Received November 4, 2013; revised April 15, 2014.

\section{Author information}

István Heckenberger, FB Mathematik und Informatik, Philipps-Universität Marburg, Hans-Meerwein-Straße, 35032 Marburg, Germany.

E-mail: heckenberger@mathematik . uni-marburg.de

Leandro Vendramin, FB Mathematik und Informatik, Philipps-Universität Marburg, Hans-Meerwein-Straße, 35032 Marburg, Germany.

E-mail: lvendramin@dm.uba.ar 